\title{
Some Results on Optimal Dividend Problem in Two Risk Models
}

\author{
Shuaiqi Zhang \\ School of Mathematical Science and Computing Technology, Central South University, Changsha, China \\ Email: shuaiqiz@yahoo.com.cn
}

\begin{abstract}
The compound Poisson risk model and the compound Poisson risk model perturbed by diffusion are considered in the presence of a dividend barrier with solvency constraints. Moreover, it extends the known result due to [1]. Ref. [1] finds the optimal dividend policy is of a barrier type for a jump-diffusion model with exponentially distributed jumps. In this paper, it turns out that there can be two different solutions depending on the model's parameters. Furthermore, an interesting result is given: the proportional transaction cost has no effect on the dividend barrier. The objective of the corporation is to maximize the cumulative expected discounted dividends payout with solvency constraints before the time of ruin. It is well known that under some reasonable assumptions, optimal dividend
\end{abstract} strategy is a barrier strategy, i.e., there is a level $b_{1}\left(b_{2}\right)$ so that whenever surplus goes above the level $b_{1}\left(b_{2}\right)$, the excess is paid out as dividends. However, the optimal level $b_{1}\left(b_{2}\right)$ may be unacceptably low from a solvency point of view. Therefore, some constraints should imposed on an insurance company such as to pay out dividends unless the surplus has reached a level $b_{c}^{1}>b_{1}\left(b_{c}^{2}>b_{2}\right)$. We show that in this case a barrier strategy at $b_{c}^{1}\left(b_{c}^{2}\right)$ is optimal.

Index Terms-optimal dividend, solvency constraints, compound Poisson risk model, compound Poisson risk model perturbed by diffusion

\section{INTRODUCTION}

The optimal dividend problem pioneered in [2]. Since then, there has been a wave of research focusing on finding the dividend payment strategy for the diffusion model and the compound Poisson risk model. In the present papers, the authors provided the solutions of the optimization problems where the companies control their risk before the cash reserves hit or fall below 0 .

For the optimal dividend problem of the compound Poisson risk model perturbed by diffusion, many papers focus on integro-differential equations for the expected discounted penalty function, as well as some ruin related functional such as the probability of ultimate ruin, the time of ruin and the surplus before ruin and the deficit at ruin. In these papers, the authors considered the problems

Footnotes:

Manuscript received July 1, 2010; revised September 1, 2010; accepted September 20, 2010.

Corresponding author: Shuaiqi Zhang within the framework that the dividend strategy is the socalled threshold dividend strategy or the barrier strategy or the multi-layer strategy. Ref.[1] studied the optimality of the dividend policy for the jump diffusion process. In this paper, some further results on the optimal dividend strategy are obtained in section III. Specifically, it turns out that there can be essentially two different solutions depending on the model's parameters. If $-\left(\frac{1}{2} \varepsilon^{2} \beta+c\right)+\beta \geq 0$, the initial capital should be paid out as dividend immediately, and the ruin occurs. If $-\left(\frac{1}{2} \varepsilon^{2} \beta+c\right)+\beta<0$, the optimal strategy is to pay everything in excess of the optimal dividend barrier, whenever the reserve reaches the barrier, and the process continues. The paper also extends the controlled model with considering the existence of the proportional transaction costs. It is shown that the amount of the dividend increases as the proportional rate $1-k$ decreases. What is also interesting is that the dividend barrier in the solution of this problem is the same as the barrier without proportional costs. In other words, proportional costs factor has no effects on the dividend barrier.

As for the dividend problems with constraints, [3] considered diffusion process under the restrictions that that the dividends should not be paid out unless the surplus has reached a level $b_{0}>b^{*}$. It is shown that in this case a barrier strategy at $b_{0}$ is optimal. This inspires us to think a question of main interest: can we find the optimal dividend strategy of the compound Poisson risk model with solvency constraints? Or can we find the optimal dividend strategy of the compound Poisson risk model perturbed by diffusion with solvency constraints?

This paper makes the same assumptions as in [3], but differently formulated because of the model we discuss are the compound Poisson risk model and compound Poisson risk model perturbed by diffusion. The objective is to maximize the discounted dividend payment with solvency constrains before the time of ruin. We solve the problem completely when the individual claim amounts are generally distributed, rather than exponentially distributed.

The paper is organized as follows: Section II establishes the control model. In Section III, some properties of the value function are presented. Section 
IV discusses some further results to the control problem when claims are exponentially distributed. In section V, the optimal dividend problem of the compound Poisson risk model with solvency constraints is solved. In section VI, the optimal dividend problem of the compound Poisson risk model perturbed by diffusion with solvency constraints is considered.

\section{Formulation OF THE CONTROL Problems}

To give a rigorous mathematical formulation of the optimization problem, we start with a filtered probability space $\left\{\Omega, \mathrm{F},\left\{\mathrm{F}_{t}\right\}_{t \geq 0}, \mathrm{P}\right\}$. We assume that in the absence of dividends, the compound Poisson risk model $X_{t}^{0}$ is given by

$$
X_{t}^{a}=x+c t-\sum_{i=1}^{N(t)} Y_{i}
$$

and the compound Poisson risk model perturbed by a diffusion is given by

$$
X_{t}^{b}=x+c t-\sum_{i=1}^{N(t)} Y_{i}+\varepsilon W_{t}
$$

where $x \geq 0$ is the initial reserve, $c>0$ is the premium rate, $\{N(t)\}$ is a Poisson process with intensity $\lambda>0$ and $\left\{Y_{i}\right\}$ is an i.i.d. sequence of strictly positive random variables with distribution function $F(y)$ and probability density function $f(y)$. The claim sizes $\left\{Y_{i}\right\}$ and the claim arrival process $N(t)$ are assumed to be independent. We assume that $\mathrm{E}\left[Y_{i}\right]=\mu<\infty$. $\left\{W_{t} ; t \geq 0\right\}$ is a standard Wiener process that is independent of the aggregate claims process $S_{t}=\sum_{k=1}^{N(t)} Y_{k}$ and $\varepsilon$ is the dispersion parameter. $X_{t}^{a}\left(X_{t}^{b}\right)$ is adapted to the smallest right-continuous filtration $\left\{\mathrm{F}_{t}\right\}_{t \geq 0}$. If we want to indicate that the initial capital is $x$, we write $\mathrm{P}_{x}$ and $\mathrm{E}_{x}$ for the probability measure and the expectation, respectively. Otherwise, we dismiss the letter $X$ and write $\mathrm{P}$ and $\mathrm{E}$.

We now enrich the compound Poison risk model (the compound Poisson risk model perturbed by diffusion). We denote by $\left\{D_{t}^{1}\right\}$ the aggregate dividends paid between time zero and time $t .\left\{D_{t}^{1}\right\}\left(\left\{D_{t}^{2}\right\}\right)$ is càglàd, increasing and adapted process with $D_{0}^{1}=0\left(D_{0}^{2}=0\right)$.

The dynamics of the controlled compound Poisson risk model is given by

$$
X_{t}^{1}=x+c t-\sum_{i=1}^{N(t)} Y_{i}-D_{t}^{1}
$$

The policy $\pi_{1}$ is said to be admissible if the process $\left\{D_{t}^{1}\right\}$ is $\left\{F_{t}\right\}_{t \geq 0}$-adapted, increasing and adapted process with $D_{0}^{1}=0$. The class of all admissible controls is denoted by $\Pi_{1}$.

The time of ruin is defined by

$$
T^{\pi_{1}}:=\inf \left\{t \geq 0: X_{t}^{1}<0\right\} \text {. }
$$

With each admissible control $\pi_{1} \in \Pi_{1}$, we associate a performance functional $V^{\pi_{1}}(x)$ defined by

$$
V^{\pi_{1}}(x)=\mathrm{E}_{x}\left(\int_{0-}^{T^{\pi_{1}}} e^{-\delta t} \mathrm{~d} D_{t}^{1}\right)
$$

where $\delta$ is the discount rate. The objective is to find the control that maximizes the performance index. We define the value function $V^{1}$ by

$$
V^{1}(x):=\sup \left\{V^{\pi_{1}}(x) ; \pi_{1} \in \Pi_{1}\right\} \text {. }
$$

The optimal policy $\pi_{1}^{*}$ is a policy for which the following equality is true:

$$
V^{1}(x)=V^{\pi_{1}^{*}}(x)
$$

If the company is not be allowed to pay dividends unless the surplus is more than $b_{c}^{1}$. Mathematically such restriction can be written as

$$
\int_{0-}^{T^{\pi_{1}}-} e^{-\delta t} I_{\left\{X_{t^{-}}^{\pi_{1}}<b_{c}^{1}\right\}} \mathrm{d} D_{t}^{1}=0
$$

If we impose restrictions of the form that no dividends are allowed to be paid out unless surplus is at least $b_{c}^{1}$, and the surplus immediately after payment can not be below $b_{c}^{1}$. In this case, we denote admissible strategy by $\Pi_{1}^{c}$. We define the performance index by

$$
V^{\pi_{1}^{c}}(x)=\mathrm{E}_{x}\left(\int_{0-}^{T_{1}^{\pi_{1}}} e^{-\delta t} \mathrm{~d} D_{t}^{1, c}\right)
$$

The value function is defined by

$$
V_{c}^{1}(x):=\sup \left\{V^{\pi_{1}^{c}}(x) ; \pi_{c}^{1} \in \Pi_{1}^{c}\right\}
$$

The optimal policy $\pi_{c}^{1^{*}}$ is a policy for which the following equality is true:

$$
V_{c}^{1}(x)=V^{\pi_{c}^{1^{*}}}(x) .
$$

We now enrich the compound Poisson risk model perturbed by diffusion. We denote by $\left\{D_{t}^{2}\right\}$ the aggregate dividends paid between time zero and time $t .\left\{D_{t}^{2}\right\}$ is càglàd, increasing and adapted process with $D_{0}^{2}=0$.

The dynamics of the controlled compound Poisson risk model perturbed by diffusion is given by

$$
X_{t}^{2}=x+c t-\sum_{i=1}^{N(t)} Y_{i}+\varepsilon W_{t}-D_{t}^{2}
$$


The policy is said to be admissible if the process $\left\{D_{t}^{2}\right\}$ is $\left\{\mathrm{F}_{t}\right\}_{t \geq 0}$-adapted, increasing and adapted process with $D_{0}^{2}=0$. The class of all admissible controls is denoted by $\Pi_{2}$.

The time of ruin is defined by

$$
T^{\pi_{2}}:=\inf \left\{t \geq 0: X_{t}^{2}<0\right\} .
$$

With each admissible control $\pi_{2} \in \Pi_{2}$, we associate a performance functional $V^{\pi_{2}}(x)$ defined by

$$
V^{\pi_{2}}(x)=\mathrm{E}_{x}\left(\int_{0-}^{T^{\pi_{2}}} e^{-\delta t} \mathrm{~d} D_{t}^{2}\right) .
$$

The value function $V^{2}(x)$ by

$$
V^{2}(x):=\sup \left\{V^{\pi_{2}}(x) ; \pi_{2} \in \Pi_{2}\right\} .
$$

The optimal policy $\pi_{2}^{*}$ is a policy for which the following equality is true:

$$
V^{2}(x)=V^{\pi_{2}^{*}}(x)
$$

If the company is not be allowed to pay dividends unless the surplus is more than $b_{c}^{2}$. Mathematically such restriction can be written as

$$
\int_{0-}^{T^{\pi_{2}}-} e^{-\delta t} I_{\left\{X_{t-}^{\pi_{2}}<b_{c}^{2}\right\}} \mathrm{d} D_{t}^{2}=0
$$

If we impose restrictions of the form that no dividends are allowed to be paid out unless surplus is at least level $b_{c}^{2}$, and the surplus immediately after payment can not be below $b_{c}^{2}$. In this case, we denote admissible strategy by $\Pi_{2}^{c}$. We define the performance index by

$$
V^{\pi_{2}^{c}}(x)=\mathrm{E}_{x}\left(\int_{0-}^{T^{\pi_{2}^{c}}} e^{-\delta t} \mathrm{~d} D_{t}^{2, c}\right)
$$

The value function is defined by

$$
V_{c}^{2}(x):=\sup \left\{V^{\pi_{2}^{c}}(x) ; \pi_{c}^{2} \in \Pi_{2}^{c}\right\} .
$$

The optimal policy $\pi_{c}^{2^{*}}$ is a policy for which the following equality is true:

$$
V_{c}^{2}(x)=V^{\pi_{c}^{2 *}}(x)
$$

\section{SOME PROPERTIES OF THE VALUE FunCTION}

We start with two lemmas.

Lemma 3.1. The function $V^{1}(x)$ and $V_{c}^{1}(x)$ are bounded. Moreover,

$$
V_{c}^{1}(x) \leq V^{1}(x) \leq x+\frac{c}{\delta} .
$$

Rroof. To get the upper bound of the value function $V^{1}(x)$, we consider a strategy $\pi_{1}$ defined as follows: the initial capital $x$ is paid immediately and then the dividends are paid at rate $c$.Then,

$$
x+\mathrm{E}_{x}\left[\int_{0}^{\infty} e^{-\delta t} c \mathrm{~d} t\right]=x+\frac{c}{\delta} .
$$

This is the upper bound of any admissible strategy, i.e.,

$$
V^{1}(x) \leq x+\frac{c}{\delta} .
$$

Since $\Pi_{1}^{c}$ is a subset of $\Pi_{1}$, obviously,

$$
V_{c}^{1}(x) \leq V^{1}(x)
$$

This completes the proof.

Lemma 3.2. The function $V^{2}(x)$ is increasing with $V^{2}(x)-V^{2}(y) \geq x-y$ for $0 \leq y \leq x$. For any $x \geq 0$,

$$
V^{2}(x) \leq x+\mu+\frac{c}{\delta} \text {. }
$$

$V^{2}(x)$ is differentiable with $V^{2 \prime}(x) \geq 1$.

Rroof. For any $\varepsilon>0$, define a strategy $\pi_{2}$ satisfing $V^{\pi_{2}}(y) \geq V^{2}(y)-\varepsilon . \pi_{2}^{\prime}$ is a new strategy for $x \geq y$. While $\pi_{2}^{\prime}$ is defined as: $x-y$ is paid immediately as dividend and then the strategy $\pi_{2}$ with initial capital $y$ is followed. Therefore,

$$
V^{2}(x) \geq x-y+V^{\pi_{2}}(y) \geq x-y+V^{2}(y)-\varepsilon .
$$

From the arbitrary property of $\varepsilon$, we have $V^{2}(x)-V^{2}(y) \geq x-y$ for $0 \leq y \leq x$. In particular, $V^{2}(x)$ is increasing. It is easy to see that $V^{2 \prime}(x) \geq 1$.

Now we prove that $V^{2}(x) \leq x+\mu+\frac{c}{\delta}$.

The proof follows the same line as Proposition 3.1 in [4]. Define a stochastic process $Z_{t}$ by

$$
Z_{t}=x+c t+\varepsilon W_{t}
$$

and

$$
T:=\inf \left\{t \geq 0: Z_{t} \leq 0\right\} .
$$

We obtain

$$
\begin{aligned}
\mathrm{E}\left[\int_{0}^{T} e^{-\delta s} \mathrm{~d} Z_{s}\right] & =\mathrm{E}\left[\int_{0}^{T} e^{-\delta s} c \mathrm{~d} s\right] \\
& \leq \mathrm{E}\left[\int_{0}^{\infty} e^{-\delta s} c \mathrm{~d} s\right]=\frac{c}{\delta}
\end{aligned}
$$

From Itô formula,

$$
e^{-\delta T^{\pi_{2}}} X_{T^{\pi_{2}}}^{2}=x-\delta \int_{0}^{T^{\pi_{2}}} e^{-\delta s} X_{s}^{2} \mathrm{~d} s+\int_{0}^{T^{\pi_{2}}} e^{-\delta s} \mathrm{~d} X_{s}^{2}
$$

Since $X_{T^{\pi_{2}}}^{2} \leq 0$, it follows that

$$
\begin{aligned}
& -\mathrm{E}\left[\int_{0}^{T^{\pi_{2}}} e^{-\delta s} \mathrm{~d} X_{s}^{2}\right] \\
= & x-\mathrm{E}\left[\delta \int_{0}^{T^{\pi_{2}}} e^{-\delta s} X_{s}^{2} \mathrm{~d} s\right]-\mathrm{E}\left[e^{-\delta T^{\pi_{2}}} X_{T^{\pi_{2}}}\right] \\
\leq & x+\mu .
\end{aligned}
$$


Clearly, combining (1) and (2), we obtain

$$
\begin{aligned}
& \mathrm{E}\left[\int_{0-}^{T^{\pi_{2}}-} e^{-\delta t} \mathrm{~d} D_{t}^{2}\right] \\
\leq & \mathrm{E}\left[\int_{0-}^{T^{\pi}-} e^{-\delta t} \mathrm{~d} Z_{t}\right]-\mathrm{E}\left[\int_{0-}^{T^{\pi_{2}}-} e^{-\delta t} \mathrm{~d} X_{t}^{2}\right] \\
\leq & x+\mu+\frac{c}{\delta} .
\end{aligned}
$$

This is the upper bound.

Since $\Pi_{2}^{c}$ is a subset of $\Pi_{2}$, obviously,

$$
V_{c}^{2}(x) \leq V^{2}(x) \text {. }
$$

This completes the proof.

\section{THE SOLUTION OF THE CONTROL PROBLEM WITHOUT RESTRICTION}

The dynamic programming principle helps us to derive the Hamilton-Jacobi-Bellman (HJB) equation.

To obtain the solution, we start with the HJB equation

$$
\begin{aligned}
& \max \left\{\frac{1}{2} \varepsilon^{2} V^{\prime \prime}(x)+c V^{\prime}(x)-(\lambda+\delta) V(x)\right. \\
& \left.+\lambda \int_{0}^{x} V(x-y) \mathrm{d} F(y), 1-V^{\prime}(x)\right\}=0 .
\end{aligned}
$$

For more detailed and complete account of the derivation, we readers should consult with [1].

In this section, we assume that the individual claim amounts are exponentially distributed,

$$
f(y)=\beta e^{-\beta y}, y>0 \text {. }
$$

From some simple calculation, as in [1], the solution to the ordinary differential equation

is given by

$$
\begin{aligned}
& \frac{1}{2} \varepsilon^{2} V_{2}^{\prime \prime \prime}(x)+\left(\frac{1}{2} \varepsilon^{2} \beta+c\right) V_{2}^{\prime \prime}(x) \\
& +(c \beta-\lambda-\delta) V_{2}^{\prime}(x)-\delta \beta V_{2}(x)=0
\end{aligned}
$$

$$
V^{2}(x)=B_{1} e^{s_{1} x}+B_{2} e^{s_{2} x}+B_{3} e^{s_{3} x}
$$

where $S_{1}, S_{2}$ and $s_{3}$ are the roots of the characteristic equation

$$
\begin{gathered}
\frac{1}{2} \varepsilon^{2} x^{3}+\left(\frac{1}{2} \varepsilon^{2} \beta+c\right) x^{2}+(c \beta \\
-\lambda-\delta) x-\delta \beta=0
\end{gathered}
$$

Moreover, $s_{1}<-\beta<s_{2}<0<s_{3}$.

Substituting (4) in the first term of equation (3), we have

$$
\frac{B_{1}}{s_{1}+\beta}+\frac{B_{2}}{s_{2}+\beta}+\frac{B_{3}}{s_{3}+\beta}=0 .
$$

From $V^{2}(0)=0$, we have

$$
B_{1}+B_{2}+B_{3}=0 \text {. }
$$

So, we obtain

$$
B_{2}=\frac{\left(s_{2}+\beta\right)\left(s_{3}-s_{1}\right)}{\left(s_{3}+\beta\right)\left(s_{1}-s_{2}\right)} B_{3},
$$

$$
B_{1}=-\frac{\left(s_{1}+\beta\right)\left(s_{3}-s_{2}\right)}{\left(s_{3}+\beta\right)\left(s_{1}-s_{2}\right)} B_{3} .
$$

Furthermore, we can conclude that $B_{1}, B_{2}$ and $B_{3}$ satisfy

$$
B_{1} B_{3}<0, B_{2} B_{3}<0, B_{1} B_{2}>0 .
$$

Now, we claim that $B_{1}<0, B_{2}<0$ and $B_{3}>0$. Because if $B_{1}>0, B_{2}>0, B_{3}<0$, we have

$$
V^{2 \prime}(0)=B_{1} s_{1}+B_{2} s_{2}+B_{3} s_{3} \text {. }
$$

This contradicts with the fact that $V^{2}(x)$ is increasing. From $V^{2 \prime}(b)=1$, we have

$$
B_{1} s_{1} e^{s_{1} b}+B_{2} s_{2} e^{s_{2} b}+B_{3} s_{3} e^{s_{3} b}=1 .
$$

Combining with the (6) and (7), we have

$$
\begin{aligned}
B_{1}= & -\frac{\left(s_{1}+\beta\right)\left(s_{3}-s_{2}\right)}{\left(s_{3}+\beta\right)\left(s_{1}-s_{2}\right)}\left\{-\frac{\left(s_{1}+\beta\right)\left(s_{3}-s_{2}\right)}{\left(s_{3}+\beta\right)\left(s_{1}-s_{2}\right)} s_{1} e^{s_{1} b}\right. \\
& \left.+\frac{\left(s_{2}+\beta\right)\left(s_{3}-s_{1}\right)}{\left(s_{3}+\beta\right)\left(s_{1}-s_{2}\right)} s_{2} e^{s_{2} b}+s_{3} e^{s_{3} b}\right\}^{-1}, \\
B_{2}= & \frac{\left(s_{2}+\beta\right)\left(s_{3}-s_{1}\right)}{\left(s_{3}+\beta\right)\left(s_{1}-s_{2}\right)}\left\{-\frac{\left(s_{1}+\beta\right)\left(s_{3}-s_{2}\right)}{\left(s_{3}+\beta\right)\left(s_{1}-s_{2}\right)} s_{1} e^{s_{1} b}\right. \\
& \left.+\frac{\left(s_{2}+\beta\right)\left(s_{3}-s_{1}\right)}{\left(s_{3}+\beta\right)\left(s_{1}-s_{2}\right)} s_{2} e^{s_{2} b}+s_{3} e^{s_{3} b}\right\}^{-1}, \\
B_{3}=\{ & -\frac{\left(s_{1}+\beta\right)\left(s_{3}-s_{2}\right)}{\left(s_{3}+\beta\right)\left(s_{1}-s_{2}\right)} s_{1} e^{s_{1} b}+\frac{\left(s_{2}+\beta\right)\left(s_{3}-s_{1}\right)}{\left(s_{3}+\beta\right)\left(s_{1}-s_{2}\right)} s_{2} e^{s_{2} b} \\
& \left.+S_{3} e^{s_{3} b}\right\}^{-1} .
\end{aligned}
$$

From $V^{2 \prime \prime}(b)=0$, we have

$$
\begin{aligned}
& -\frac{\left(s_{1}+\beta\right)\left(s_{3}-s_{2}\right)}{\left(s_{3}+\beta\right)\left(s_{1}-s_{2}\right)} s_{1}^{2} e^{s_{1} b}+\frac{\left(s_{2}+\beta\right)\left(s_{3}-s_{1}\right)}{\left(s_{3}+\beta\right)\left(s_{1}-s_{2}\right)} s_{2}^{2} e^{s_{2} b} \\
& +s_{3}^{2} e^{s_{3} b}=0 .
\end{aligned}
$$

Denote the left hand side of the equation (11) by $h(z)$,

$$
\begin{gathered}
h(z)=-\frac{\left(s_{1}+\beta\right)\left(s_{3}-s_{2}\right)}{\left(s_{3}+\beta\right)\left(s_{1}-s_{2}\right)} s_{1}^{2} e^{s_{1} z} \\
+\frac{\left(s_{2}+\beta\right)\left(s_{3}-s_{1}\right)}{\left(s_{3}+\beta\right)\left(s_{1}-s_{2}\right)} s_{2}^{2} e^{s_{2} z}+s_{3}^{2} e^{s_{3} z},
\end{gathered}
$$

Now we differentiate (12) with respect to $Z$ and get

$$
\begin{aligned}
h^{\prime}(z)= & -\frac{\left(s_{1}+\beta\right)\left(s_{3}-s_{2}\right)}{\left(s_{3}+\beta\right)\left(s_{1}-s_{2}\right)} s_{1}^{3} e^{s_{1} z} \\
& +\frac{\left(s_{2}+\beta\right)\left(s_{3}-s_{1}\right)}{\left(s_{3}+\beta\right)\left(s_{1}-s_{2}\right)} s_{2}^{3} e^{s_{2} z}+s_{3}^{3} e^{s_{3} z}>0 .
\end{aligned}
$$

In view of the calculation

$$
\lim _{z \rightarrow-\infty} h(z)=-\infty \text { and } \lim _{z \rightarrow+\infty} h(z)=+\infty,
$$


we can see that there exists a unique $b$ s.t. $h(b)=0$. If $b>0$, such $b$ is the optimal dividend barrier. If $b \leq 0$, then 0 is the dividend barrier.

Now we calculate

$$
\begin{aligned}
h(0)= & -\frac{\left(s_{1}+\beta\right)\left(s_{3}-s_{2}\right)}{\left(s_{3}+\beta\right)\left(s_{1}-s_{2}\right)} s_{1}^{2}+\frac{\left(s_{2}+\beta\right)\left(s_{3}-s_{1}\right)}{\left(s_{3}+\beta\right)\left(s_{1}-s_{2}\right)} s_{2}^{2} \\
& +s_{3}^{2} \\
= & \frac{\left(s_{1}-s_{3}\right)\left(s_{2}-s_{3}\right)\left(s_{1}+s_{2}+s_{3}+\beta\right)}{s_{3}+\beta} .
\end{aligned}
$$

From (5), we have

$$
s_{1}+s_{2}+s_{3}=-\left(\frac{1}{2} \varepsilon^{2} \beta+c\right) .
$$

So, the fact $s_{1}+s_{2}+s_{3}+\beta=-\left(\frac{1}{2} \varepsilon^{2} \beta+c\right)+\beta$ implies

$$
\left\{\begin{array}{l}
b \leq 0 \Leftrightarrow h(0) \geq 0 \Leftrightarrow-\left(\frac{1}{2} \varepsilon^{2} \beta+c\right)+\beta \geq 0, \\
b>0 \Leftrightarrow h(0)<0 \Leftrightarrow-\left(\frac{1}{2} \varepsilon^{2} \beta+c\right)+\beta<0 .
\end{array}\right.
$$

If $b \leq 0$, the optimal dividend policy is that at time 0 , the initial capital $x$ should be paid out. If $b>0$, whenever surplus goes above the level $b$, the excess is paid out as dividends.

From the above analysis, the solution to the problem is given by follows.

$$
\begin{aligned}
& \text { If }-\left(\frac{1}{2} \varepsilon^{2} \beta+c\right)+\beta \geq 0, \\
& \qquad V_{2}(x)=x . \\
& \text { If }-\left(\frac{1}{2} \varepsilon^{2} \beta+c\right)+\beta<0, \\
& \qquad V^{2}(x)=\left\{\begin{array}{cc}
B_{1} e^{s_{1} x}+B_{2} e^{s_{2} x}+B_{3} e^{s_{3} x}, & 0 \leq x<b, \\
x-b+V_{2}(b), & x>b .
\end{array}\right.
\end{aligned}
$$

where $S_{1}, S_{2}$ and $S_{3}$ are the roots of the characteristic equation (5), $B_{1}, B_{2}$ and $B_{3}$ are determined by (8), (9) and (10), $b$ is determined by (11).

The above model makes stylized assumption that there are no transaction costs. Strictly speaking, the assumption is not satisfied in real applications. Now, we study the model where the transaction costs are proportional. If the company pays $l$ as dividends, then the shareholders can get $k l, k<1$. In the meanwhile, $(1-k) l$ is the proportional transaction costs. In this case, we denote the value function by $W(x)$. Repeat the procedure above, we obtain

$$
W(x)=k V^{2}(x) .
$$

Now we investigate how $W(x)$ depends on $k$.

$$
\begin{aligned}
\frac{\mathrm{d}}{\mathrm{d} k} W(x, k) & =B_{1} e^{s_{1} x}+B_{2} e^{s_{2} x}+B_{3} e^{s_{3} x} \\
& =V^{2}(x) \geq 0 .
\end{aligned}
$$

Note that the dividend barrier in the solution of this problem is the same as the barrier of the problem without proportional costs. In other words, proportional costs factor $k$ has no effects on the dividend barrier.

\section{THE SOLUTION OF THE CONTROL PROBLEM FOR THE CLASSICAL RISK MODEL WITH SOLVENCY CONSTRAINTS}

An interesting discovery in [5] is the expression of the value function. That is,

$$
V^{1}(x, b)=\frac{h(x)}{h^{\prime}(b)}, 0 \leq x \leq b .
$$

In what follows, we use this factorization formula that facilitates solving the optimal dividend problem with solvency constraints. The idea is inspired by [6].

Theorem 5.1. If $h(x)$ is convex for $x \geq b_{c}^{1}$,

$$
\begin{aligned}
& c V_{c}^{1^{\prime}}(x)-(\lambda+\delta) V_{c}^{1}(x) \\
& +\lambda \int_{0}^{x} V_{c}^{1}(x-y) \mathrm{d} F(y) \leq 0 .
\end{aligned}
$$

Proof. For any $x \geq b_{1}, b_{1}<z<x$,

$$
\begin{aligned}
& c V_{x}^{1^{\prime}}(z)-(\lambda+\delta) V_{x}^{1}(z) \\
& +\lambda \int_{0}^{x} V_{x}^{1}(z-y) \mathrm{d} F(y)=0,
\end{aligned}
$$

and

$$
\begin{aligned}
& c V_{b^{1}}^{1^{\prime}}(z)-(\lambda+\delta) V_{b^{1}}^{1}(z)+\lambda \int_{0}^{z} V_{b^{1}}^{1}(z-y) \mathrm{d} F(y) \\
= & c\left(V_{b^{1}}^{1^{\prime}}-V_{x}^{1^{\prime}}\right)(z)-(\lambda+\delta)\left(V_{b^{1}}^{1}-V_{x}^{1}\right)(z) \\
& +\lambda \int_{0}^{z}\left(V_{b^{1}}^{1}-V_{x}^{1}\right)(z-y) \mathrm{d} F(y) \\
= & c\left(V_{b^{1}}^{1^{\prime}}-V_{x}^{1^{\prime}}\right)(z)-\delta\left(V_{b^{1}}^{1}-V_{x}^{1}\right)(z) \\
& -\lambda \int_{0}^{z}\left(V_{b^{1}}^{1}-V_{x}^{1}\right)(z)-\left(V_{b^{1}}^{1}-V_{x}^{1}\right)(z-y) \mathrm{d} F(y) \\
\leq & c\left(V_{b^{1}}^{1^{\prime}}-V_{x}^{1^{\prime}}\right)(z) .
\end{aligned}
$$

In fact, $h(x)$ is convex on $\left[b_{c}^{1}, \infty\right)$, therefore,

$$
\begin{aligned}
& \left(V_{b^{1}}^{1}-V_{x}^{1}\right)^{\prime}(y)=1-\frac{h^{\prime}(y)}{h^{\prime}(x)} \geq 0, b_{1} \leq y<x . \\
& \left(V_{b^{1}}^{1}-V_{x}^{1}\right)^{\prime}(y)=\frac{h^{\prime}(y)}{h^{\prime}\left(b^{1}\right)}-\frac{h^{\prime}(y)}{h^{\prime}(x)} \geq 0, y<b_{1} .
\end{aligned}
$$

Letting $Z \rightarrow X$ on both sides of (13), we obtain

$$
\begin{aligned}
& c V_{b^{1}}^{1^{\prime}}(x)-(\lambda+\delta) V_{b^{1}}^{1}(x) \\
& +\lambda \int_{0}^{x} V_{b^{1}}^{1}(x-y) \mathrm{d} F(y) \leq 0 .
\end{aligned}
$$


Theorem 5.2 Assume the dividend policy $\left\{D_{t}^{1}\right\}$ has to satisfy the solvency constraints for some positive $b_{c}^{1}$. $b^{1}$ is the optimal barrier without restriction. Assume that $h(x)$ is convex for $x \geq b^{1}$. Then

(a) If $b_{c}^{1} \leq b^{1}$, the optimal policy is to use the barrier strategy at $b^{1}$.

(b) If $b_{c}^{1}>b^{1}$, the optimal policy is to use the barrier strategy at $b_{c}^{1}$.

Proof. Part (a) is obvious since the optimal policy is feasible under the constraint.

(b) For any $b_{4}>b_{3} \geq b_{c}^{1}$, we have

$$
V^{1}\left(x, b_{4}\right)<V^{1}\left(x, b_{3}\right)
$$

Indeed, for $x>b_{4}>b_{3}$,

$$
\begin{gathered}
V^{1}\left(x, b_{3}\right)=x-b_{3}+\frac{h\left(b_{3}\right)}{h^{\prime}\left(b_{3}\right)}, \\
V^{1}\left(x, b_{4}\right)=x-b_{4}+\frac{h\left(b_{4}\right)}{h^{\prime}\left(b_{4}\right)}, \\
V^{1}\left(x, b_{3}\right)-V^{1}\left(x, b_{4}\right)=b_{4}-b_{3}+\frac{h\left(b_{3}\right)}{h^{\prime}\left(b_{3}\right)}-\frac{h\left(b_{4}\right)}{h^{\prime}\left(b_{4}\right)} .
\end{gathered}
$$

Let

$$
j(y)=y-b_{3}+\frac{h\left(b_{3}\right)}{h^{\prime}\left(b_{3}\right)}-\frac{h(y)}{h^{\prime}\left(b_{4}\right)} .
$$

By simple calculation, $j\left(b_{3}\right) \geq 0$ and $j^{\prime}(y) \geq 0$ for $b_{4} \geq y \geq b_{3}$. Hence,

$$
V^{1}\left(x, b_{3}\right)-V^{1}\left(x, b_{4}\right)=j\left(b_{4}\right) \geq 0 .
$$

For $b_{3}<x \leq b_{4}$,

$$
\begin{aligned}
V^{1}\left(x, b_{3}\right) & =x-b_{3}+\frac{h\left(b_{3}\right)}{h^{\prime}\left(b_{3}\right)}, \\
V^{1}\left(x, b_{4}\right) & =\frac{h(x)}{h^{\prime}\left(b_{4}\right)}, \\
V^{1}\left(x, b_{3}\right)-V^{1}\left(x, b_{4}\right) & =x-b_{3}+\frac{h\left(b_{3}\right)}{h^{\prime}\left(b_{3}\right)}-\frac{h(x)}{h^{\prime}\left(b_{4}\right)} \\
& =j(x) \geq 0 .
\end{aligned}
$$

For $x \leq b_{3}<b_{4}$,

$$
\begin{gathered}
V^{1}\left(x, b_{3}\right)=\frac{h(x)}{h^{\prime}\left(b_{3}\right)}, \\
V^{1}\left(x, b_{4}\right)=\frac{h(x)}{h^{\prime}\left(b_{4}\right)}, \\
V^{1}\left(x, b_{3}\right)-V^{1}\left(x, b_{4}\right) \geq 0 .
\end{gathered}
$$

So,

$$
V_{c}^{1}(x)=\max _{b \geq b_{1}} V^{1}(x, b), \forall x \geq 0 .
$$

If the barrier strategy $b_{c}^{1}$ is used,

$$
\begin{aligned}
& c V_{c}^{1^{\prime}}(x)-(\lambda+\delta) V_{c}^{1}(x) \\
& \quad+\lambda \int_{0}^{x} V_{c}^{1}(x-y) \mathrm{d} F(y)=0,0 \leq x<b_{c}^{1} .
\end{aligned}
$$

From Theorem 5.1,

$$
\begin{aligned}
& c V_{c}^{1^{\prime}}(x)-(\lambda+\delta) V_{c}^{1}(x) \\
& \quad+\lambda \int_{0}^{x} V_{c}^{1}(x-y) \mathrm{d} F(y) \leq 0, \quad x \geq b_{c}^{1} . \\
& V_{c}^{1}\left(X_{t \wedge T^{\pi}}^{1}\right) e^{-\delta\left(t \wedge T^{\pi}\right)} \\
& =V_{c}^{1}(x)+\int_{0}^{t \wedge T^{\pi}} e^{-\delta s}\left[c V_{c}^{1^{\prime}}\left(X_{s}^{1}\right)-(\lambda+\delta) V_{c}^{1}\left(X_{s}^{1}\right)\right. \\
& \left.+\lambda \int_{0}^{X_{s-}^{1}} V_{c}^{1}\left(X_{s-}^{1}-y\right) \mathrm{d} F(y)\right] \mathrm{d} s \\
& -\int_{0}^{t \wedge T^{\pi}} e^{-\delta s} V_{c}^{1^{\prime}}\left(X_{s}^{1}\right) \mathrm{d} D_{s}^{c} \\
& +\sum_{0<s \leq t \wedge T^{\pi}} e^{-\delta s}\left[V_{c}^{1}\left(X_{s-}^{1}-\Delta D_{s}^{1}\right)-V_{c}^{1}\left(X_{s-}^{1}\right)\right] .(14)
\end{aligned}
$$

Furthermore, applying the barrier strategy $b_{c}^{1}$,

$$
\begin{gathered}
V_{c}^{1}\left(X_{s-}^{1}-\Delta D_{s}^{1}\right)-V_{c}^{1}\left(X_{s-}^{1}\right)=-\Delta D_{s}^{1} . \\
-\int_{0}^{t \wedge T^{\pi}} e^{-\delta s} V_{c}^{1^{\prime}}\left(X_{s}^{1}\right) \mathrm{d} D_{s}^{c}=-\int_{0}^{t \wedge T^{\pi}} e^{-\delta s} \mathrm{~d} D_{s}^{c}
\end{gathered}
$$

Therefore, taking expectation in (14), we have

$$
\mathrm{E} \int_{0}^{t \wedge T^{\pi}} e^{-\delta s} \mathrm{~d} D_{t}^{1, c} \leq V_{c}^{1}(x)-V_{c}^{1}\left(X_{t \wedge T^{\pi}}^{1}\right) e^{-\delta\left(t \wedge T^{\pi}\right)} .
$$

Now letting $t \rightarrow \infty$, it follows from bounded convergence theorem that

$$
\mathrm{E} \int_{0}^{t \wedge T^{\pi}} e^{-\delta s} \mathrm{~d} D_{t}^{1, c} \leq V_{c}^{1}(x) .
$$

The proof is completed.

\section{THE SOLUTION OF THE CONTROL PROBLEM FOR THE CLASSICAL RISK MODEL PERTURBED BY DIFFUSION WITH SOLVENCY CONSTRAINTS}

In this section, we can use the similar argument as section $\mathrm{V}$.

Theorem 6.1. If $h(x)$ is convex for $x \geq b_{c}^{2}$,

$$
\begin{gathered}
\frac{1}{2} \varepsilon^{2} V_{c}^{2^{\prime \prime}}\left(X_{s}^{\pi}\right)+c V_{c}^{2^{\prime}}(x)-(\lambda+\delta) V_{c}^{2}(x) \\
+\lambda \int_{0}^{x} V_{c}^{2}(x-y) \mathrm{d} F(y) \leq 0 .
\end{gathered}
$$

The proof is similar to the proof of Theorem 5.1.

Thereom 6.2. Assume the dividend policy $\left\{D_{t}^{2}\right\}$ has to satisfy the solvency constraints for some positive $b_{c}^{2}$. $b^{2}$ is the optimal barrier without restriction. Assume that $h(x)$ is convex for $x \geq b^{2}$. Then 
(a) If $b_{c}^{2} \leq b^{2}$, the optimal policy is to use the barrier strategy at $b^{2}$.

(b) If $b_{c}^{2}>b^{2}$, the optimal policy is to use the barrier strategy at $b_{c}^{2}$.

Proof. Part (a) is obvious since the optimal policy is feasible under the constraint.

(b). As the corresponding part of Theorem 5.2, we have

$$
V_{c}^{2}(x)=\max _{b \geq b_{2}} V^{2}(x, b), \forall x \geq 0 .
$$

If the barrier strategy $b_{c}^{2}$ is used,

$$
\begin{aligned}
& \frac{1}{2} \varepsilon^{2} V_{c}^{2 \prime \prime}(x)+c V_{c}^{2 \prime}(x)-(\lambda+\delta) V_{c}^{2}(x) \\
& \quad+\lambda \int_{0}^{x} V_{c}^{2}(x-y) \mathrm{d} F(y)=0,0 \leq x<b_{c}^{2} .
\end{aligned}
$$

From Theorem 6.1,

$$
\begin{aligned}
& \frac{1}{2} \varepsilon^{2} V_{c}^{2 \prime \prime}(x)+c V_{c}^{2 \prime}(x)-(\lambda+\delta) V_{c}^{2}(x) \\
& \quad+\lambda \int_{0}^{x} V_{c}^{2}(x-y) \mathrm{d} F(y) \leq 0 . \quad x \geq b_{c}^{2} . \\
& V_{c}^{2}\left(X_{t \wedge T^{\pi}}^{2}\right) e^{-\delta\left(t \wedge T^{\pi}\right)} \\
& =V_{c}^{2}(x)+\int_{0}^{t \wedge T^{\pi}} e^{-\delta s}\left[\frac{1}{2} \varepsilon^{2} V_{c}^{2^{\prime \prime}}\left(X_{s}^{2}\right)+c V_{c}^{2 \prime}\left(X_{s}^{2}\right)\right. \\
& -(\lambda+\delta) V_{c}^{2}\left(X_{s}^{2}\right)+\lambda \int_{0}^{X_{s-}^{2}} V_{c}^{2}\left(X_{s-}^{2}\right. \\
& -y) \mathrm{d} F(y)] \mathrm{d} s+\int_{0}^{t \wedge T^{\pi}} e^{-\delta s} V_{c}^{2 \prime}\left(X_{s}^{2}\right) \mathrm{dW} \\
& -\int_{0}^{t \wedge T^{\pi}} e^{-\delta s} V_{c}^{2^{\prime}}\left(X_{s}^{2}\right) \mathrm{d} D_{s}^{c} \\
& +\sum_{0<s \leq t \wedge T^{\pi}} e^{-\delta s}\left[V_{c}^{2}\left(X_{s-}^{2}-\Delta D_{s}^{2}\right)-V_{c}^{2}\left(X_{s-}^{2}\right)\right] .(15)
\end{aligned}
$$

Furthermore, applying the barrier strategy $b_{c}^{1}$,

$$
\begin{gathered}
V_{c}^{2}\left(X_{s-}^{2}-\Delta D_{s}^{2}\right)-V_{c}^{2}\left(X_{s-}^{\pi}\right)=-\Delta D_{s}^{2} . \\
-\int_{0}^{t \wedge T^{\pi}} e^{-\delta s} V_{c}^{2 \prime}\left(X_{s}^{2}\right) \mathrm{d} D_{s}^{c}=-\int_{0}^{t \wedge T^{\pi}} e^{-\delta s} \mathrm{~d} D_{s}^{c} .
\end{gathered}
$$

Therefore, taking expectation in (14), we have

$$
\mathrm{E} \int_{0}^{t \wedge T^{\pi}} e^{-\delta s} \mathrm{~d} D_{t}^{2, c} \leq V_{c}^{2}(x)-V_{c}^{2}\left(X_{t \wedge T^{\pi}}^{2}\right) e^{-\delta\left(t \wedge T^{\pi}\right)} .
$$

Now letting $t \rightarrow \infty$, it follows from bounded convergence theorem that

$$
\mathrm{E} \int_{0}^{t \wedge T^{\pi}} e^{-\delta s} \mathrm{~d} D_{t}^{2, c} \leq V_{c}^{2}(x)
$$

The proof is completed.

\section{REFERENCES}

[1] M. Belhaj, "Optimal dividend payments when cash reserves follow a jump-diffusion process," Math. Finance, vol. 20, pp. 313-325, 2010.

[2] B. deFinetti, "Su un'impostazione alternativa della teoria collettiva del rischio," Transactions of the XVth International Congress of Actuaries,vol.2,pp.433-443,1957.

[3] J. Paulsen, "Optimal dividend payouts for diffusions with solvency constraints, ” Finance Stochast. vol. 7, pp. 457473, 2003.

[4] A. Cadenillas, T. Choulli,, M. Taksar, ,and L..Zhang, "Classical and impulse stochastic control for the optimization of the dividend and risk policies of an insurance firm,” Math. Finance, vol. 16, pp. 181-202, 2006.

[5] H. Bühlmann, Mathematical Methods in Risk Thoery, New York: Springer-Verlag, 1970.

[6] Y. Fang. "Optimal dividend problem for the risk model with constant interest rate,” Doctoral Dissertation, 2009.

[7] H.U. Gerber, and E.S.W. Shiu, "Optimal dividends: analysis with Brownian motion,” N. Am. Actuar. J. vol.8, pp.1-20. 2004.

[8] D.C.M. Dickson, and H.R. Waters, "Some optimal dividends problems," Astin Bulletin, vol. 34 , pp.49-74. 2004.

[9] B. Hojgaard, and M. Taksar, "Controlling risk exposure and dividends pay-out schemes: Insurance company example,” Math. Finance. vol. 9, pp.153-182. 1999.

[10] H.U. Gerber, and E.S.W. Shiu, "On optimal dividend strategies in the Compound Poisson model," N. Am. Actuar. J. vol.10 , pp. 76-93, 2006.

[11] H. Gerber, and Shiu, E., 2006. "On optimal dividends: From refraction to refraction,” J. Comp. Appl. Math .vol. 186, pp.4-22, 2006.

Shuaiqi Zhang: (1983-) doctorial student of probability theory, Central South University. 